\title{
High mobility epitaxial graphene devices via aqueous-ozone processing
}

\author{
Tom Yager, ${ }^{1, a)}$ Matthew J. Webb, ${ }^{2, a)}$ Helena Grennberg, ${ }^{2}$ Rositsa Yakimova, ${ }^{3}$ \\ Samuel Lara-Avila, ${ }^{1}$ and Sergey Kubatkin ${ }^{1}$ \\ ${ }^{1}$ Department of Microtechnology and Nanoscience, Chalmers University of Technology, Göteborg S-412 96, \\ Sweden \\ ${ }^{2}$ Department of Chemistry-BMC, Uppsala University, Box 576, Uppsala S-751 23, Sweden \\ ${ }^{3}$ Department of Physics, Chemistry and Biology (IFM), Linköping University, Linköping S-581 83, Sweden
}

(Received 13 December 2014; accepted 30 January 2015; published online 11 February 2015)

\begin{abstract}
We find that monolayer epitaxial graphene devices exposed to aggressive aqueous-ozone processing and annealing became cleaner from post-fabrication organic resist residuals and, significantly, maintain their high carrier mobility. Additionally, we observe a decrease in carrier density from inherent strong n-type doping to extremely low p-type doping after processing. This transition is explained to be a consequence of the cleaning effect of aqueous-ozone processing and annealing, since the observed removal of resist residuals from $\mathrm{SiC} / \mathrm{G}$ enables the exposure of the bare graphene to dopants present in ambient conditions. The resulting combination of charge neutrality, high mobility, large area clean surfaces, and susceptibility to environmental species suggest this processed graphene system as an ideal candidate for gas sensing applications. (C) 2015 AIP Publishing LLC. [http://dx.doi.org/10.1063/1.4907947]
\end{abstract}

Graphene, a single atomic carbon layer, is exceptionally sensitive to the presence of chemical species at its surface. This inherent sensitivity of graphene offers the capability of detecting the presence of surface adsorbates, even single molecules, ${ }^{1}$ through changes in electronic properties. ${ }^{2-4}$ However, for graphene devices, this sensitivity also presents a challenge, since device performance and reproducibility are heavily influenced by both the chemical environment and by contaminant species at the graphene surface. In particular, residuals of organic polymeric species left over from resist-based lithography can limit surface sensitive applications as well as degrade electronic device performance by introducing inhomogeneous doping profiles and scattering. In addition, resist residuals are known to contribute to poor interfaces during device fabrication and inhibit nanoscale microscopy.

A common method for removing contaminants in semiconductor technology involves ozone, ${ }^{5-7}$ generated by irradiation of molecular oxygen with ultraviolet light (UV) to decompose organic molecules at the surface of electronic materials. Similar ozone-based methodologies involving graphene devices have resulted in an initial, unstable, p-type doping effect ${ }^{8-12}$ and subsequent decomposition of graphene. ${ }^{13}$ Alternative graphene-cleaning methods include current annealing $^{14}$ and contact mode atomic force microscopy (AFM), ${ }^{15}$ however, these are unsuitable for wafer-scale applications. A promising route for ensuring a clean post-fabrication graphene surface is to employ specific polymer resists, followed by high temperature thermal annealing. ${ }^{16}$ Although this method is compatible with wafer scale processing, its impact on electronic transport properties of graphene, such as carrier mobility and concentration, has not been reported.

In this study, we have investigated the effect of an aqueous-ozone based protocol on the surface morphology and transport properties of epitaxial graphene devices on

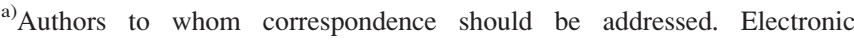
addresses: yager@chalmers.se and matthew.webb@cantab.net
}

silicion carbide $(\mathrm{SiC} / \mathrm{G})$. The aggressive process, that involves immersing graphene samples in an aqueous solution of ozone followed by thermal annealing in ultrahigh vacuum (UHV), has been observed to attack silica glass and $\mathrm{Si} / \mathrm{SiO}_{2}$ substrates and requires a custom teflon reaction vessel. Yet, aqueous-ozone processing has previously been shown to be compatible with $\mathrm{SiC} / \mathrm{G}$ by means of surface characterisation techniques, ${ }^{17}$ in contrast to in situ generated ozone gas that damages graphene devices. Using AFM and temperature dependant magnetotransport, we found that monolayer epitaxial graphene devices exposed to the aggressive chemical environment became cleaner from post-fabrication organic resist residuals. Significantly, aqueous-ozone treated $\mathrm{SiC} / \mathrm{G}$ devices maintained their electronic transport performance, in terms of carrier mobility, and display a decrease in carrier density from inherent n-type doping (specific to $\mathrm{SiC} / \mathrm{G})^{18,19}$ to extremely low p-type doping after processing.

We studied Hall bar devices on $\mathrm{SiC} / \mathrm{G}$ grown on Si-face of $4 \mathrm{H}-\mathrm{SiC}$ at $T=2000^{\circ} \mathrm{C}$ and $P=1 \mathrm{~atm} \mathrm{Ar}$ (Graphensic AB). ${ }^{20}$ Devices with dimensions $W=10 \mu \mathrm{m} \times L=24 \mu \mathrm{m}$ were patterned on $\mathrm{SiC} / \mathrm{G}$ using standard electron-beam lithography, lift-off, and oxygen plasma etching. ${ }^{21}$ After patterning, the samples were encapsulated ${ }^{22}$ in PMMA resist to preserve the doping level and enable initial electrical characterization of the material in terms of carrier density and mobility before being washed in acetone and isopropanol. Following initial characterization, the samples were immersed in a Teflon vessel containing deionized water into which ozone, generated ex situ in a molecular oxygen gas stream, was bubbled through the reaction vessel for $3 \mathrm{~min}$ prior to rinsing the devices in deionized water and vacuumdrying $\left(1\right.$ millibar, $60^{\circ} \mathrm{C}$ ). The samples were then annealed $\left(500^{\circ} \mathrm{C}\right.$ ) in either UHV (sample A, $60 \mathrm{~min}$ ) or an inert gas atmosphere (sample B, $10 \mathrm{~min}$ ) before further surface and magnetotransport measurements.

Surface characterization of $\mathrm{SiC} / \mathrm{G}$ by AFM revealed that the process has a cleaning effect on the samples, by 

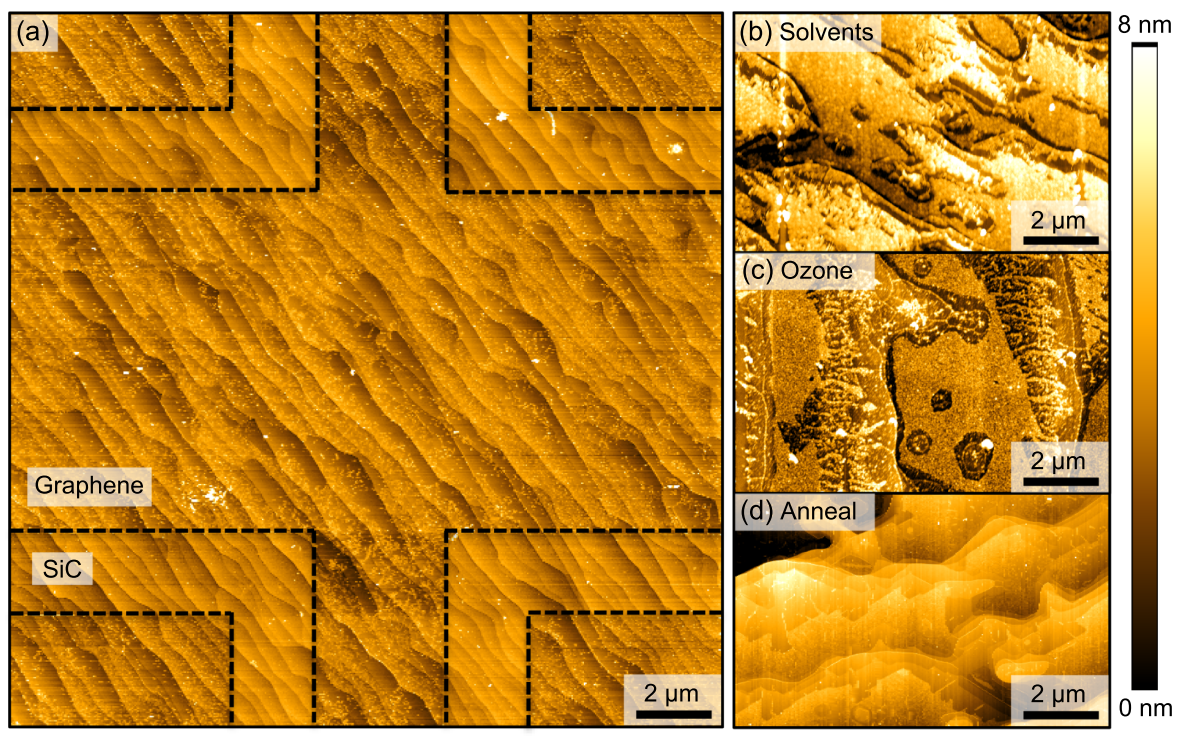

FIG. 1. Height AFM images showing each step of the aqueous-ozone and annealing process for PMMA coated $\mathrm{SiC} / \mathrm{G}$. (a) A predominantly monolayer graphene Hall bar device after combined aqueous-ozone and annealing processing, demonstrating a substantially cleaned surface. (b) A PMMA coated graphene surface after solvent cleaning with acetone and isopropanol with a few nanometers of resist residuals remaining. (c) $3 \mathrm{~min}$ aqueousozone exposed surface, after organic solvents, showing anisotropic cleaning. (d) Rapid thermal annealing (10 min, $500^{\circ} \mathrm{C}$ ) after aqueous-ozone processing leads to a cleaner surface, comparable with sample A (annealed in UHV).

removing resist residuals present on the graphene surface after microfabrication. Figure 1(a) shows a SiC/G Hall bar device (sample A) after processing. The topography of the sample, as shown by height AFM, indicates substantial cleaning to the graphene surface. In particular, the bare SiC and bilayer graphene regions (dark height contrast, confirmed by optical microscopy ${ }^{23}$ ) exhibit a very sparse coverage of resist residuals remaining at the surface.

In order to discern the cleaning contribution of each process step, we used AFM to study the topography before and after aqueous-ozone processing and then subsequently after rapid thermal annealing in an argon atmosphere (sample B). Before processing, AFM scans after conventional acetone/ isopropanol solvent washing (Figure 1(b)) indicated that several nanometers of resist residuals remained on the graphene surface. Subsequent aqueous-ozone processing (3 min) resulted in uneven cleaning, with resist residuals preferentially removed from bilayer graphene (Figure 1(c)). However, some contamination still remained on the graphene surface. After rapid thermal annealing in argon (10 $\min , 500^{\circ} \mathrm{C}$ ) the majority of resist residuals were removed and a significantly cleaner surface was exhibited (Figure 1(d)). The resulting surface of sample B closely resembled that of sample A, annealed under UHV.

The removal of resist residuals using these cleaning methods was found to be most effective for bare $\mathrm{SiC}$ and bilayer graphene. In contrast to this observation, mechanical cleaning by contact mode AFM preferentially cleans monolayer and bilayer graphene domains, but is less effective at removing resist from bare $\mathrm{SiC}^{23}$ This suggests a lower binding energy on $\mathrm{SiC}$ and bilayer graphene than for monolayer, but a low translational barrier on the graphene surface in comparison to the bare $\mathrm{SiC}$ surface.

Together with the cleaning effect on the surface of SiC/ $\mathrm{G}$, we observed a transition from n-type to low p-type doping and preservation of the carrier mobility after the aqueousozone treatment. As-grown $\mathrm{SiC} / \mathrm{G}$ samples are systematically measured by angle-resolved photoemission spectroscopy (ARPES) to exhibit strong n-type doping on the order of $\sim 10^{13}$ electrons $\mathrm{cm}^{-2}$ under UHV conditions. ${ }^{18}$ The origin of this intrinsic heavy $n$-doping is the electrostatic interaction between graphene and the $\mathrm{SiC}$ substrate via the buffer layer. ${ }^{19}$ However, when encapsulated with polymer resist ${ }^{22}$ the Hall carrier density is measured to be $\sim 3-8 \times 10^{12}$ electrons $\mathrm{cm}^{-2}$, depending on the proportion of monolayer and bilayer graphene present in the device. ${ }^{23,24}$ In this study, all polymer encapsulated devices exhibited n-type doping, obtained by low-field Hall measurements as $n=1 / \mathrm{eR}_{\mathrm{H}}=1 / \mathrm{e}\left(\mathrm{dR}_{\mathrm{xy}} / \mathrm{dB}\right) \approx 4$ $\times 10^{12}$ electrons $\mathrm{cm}^{-2}$ and Hall mobility, estimated as $\mu=\rho_{\mathrm{xx}} / \mathrm{R}_{\mathrm{H}} \approx 1500 \mathrm{~cm}^{2} \mathrm{Vs}^{-1}$ at room temperature (sample A). After processing, magnetotransport measurements revealed a change of sign in the Hall coefficient, signalling a transition from electron to p-type doping at the level of $p=4.6 \times 10^{11}$ holes $\mathrm{cm}^{-2}$ whilst the Hall mobility was maintained above $1400 \mathrm{~cm}^{2} \mathrm{Vs}^{-1}$, also at room temperature. An overview of the graphene carrier density throughout all processing steps presented in this study is shown in Figure 2(a).

The high quality and electronic integrity of the processed device were revealed from low temperature magnetotransport measurements, by a Hall mobility of $\mu=11000 \mathrm{~cm}^{2} \mathrm{~V}^{-1} \mathrm{~s}^{-1}$ and extremely low carrier density of $p=4 \times 10^{10}$ holes $\mathrm{cm}^{-2}$ at liquid helium temperature (Figure 2(b)). Subsequently, the quantum Hall effect was observed for this device at magnetic fields as low as $B=2 \mathrm{~T}$ (Figure 2(c)). Hall plateau observed at $R_{x y}= \pm \mathrm{h} /\left(2 \mathrm{e}^{2}\right)$, where $h$ is the Planck constant and $e$ is the elementary charge, are the fingerprint of monolayer graphene, ${ }^{25}$ confirming that charge transport in the processed $\mathrm{SiC} / \mathrm{G}$ is dominated by monolayer graphene, thus ruling out intercalation of chemical species at the graphene-SiC interface. ${ }^{26}$ This latter statement is supported by the strong temperature dependence of the carrier mobility, measured from room temperature down to $4 \mathrm{~K}$ (Figure 2(b)). In $\mathrm{SiC} / \mathrm{G}$, the graphene layer sits on top of an electrically insulating, graphene-like layer (buffer layer) chemically bonded to the $\mathrm{SiC}$ substrate. It has been shown that it is possible to decouple the buffer layer from the SiC substrate by intercalation of species such as hydrogen. ${ }^{26}$ In sharp contrast to our processed samples, intercalation results in a quasi-freestanding bilayer graphene on the surface of $\mathrm{SiC}$, which displays temperature independent carrier mobility and QHE plateaux sequence for bilayer graphene. $^{27}$ 

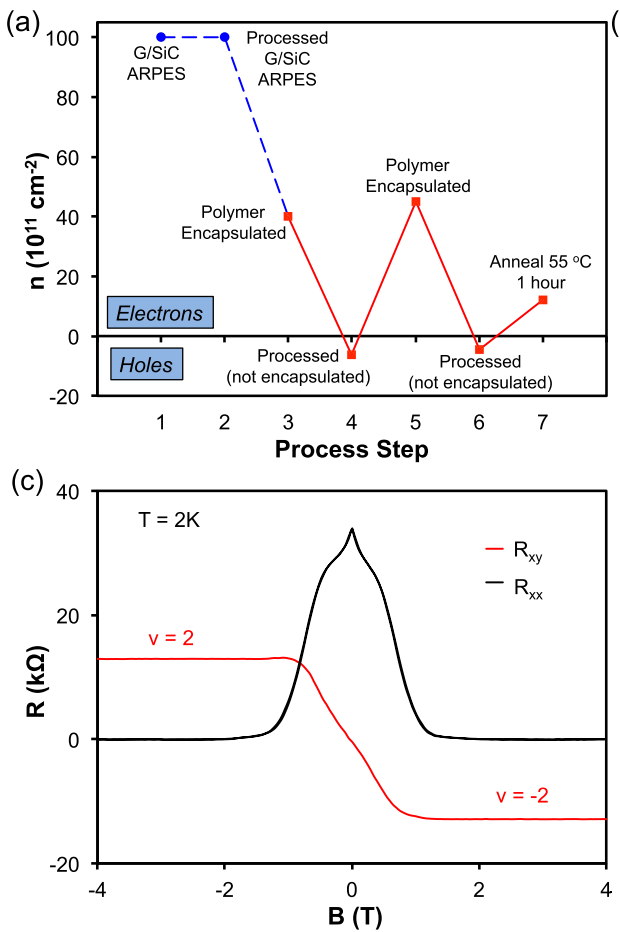

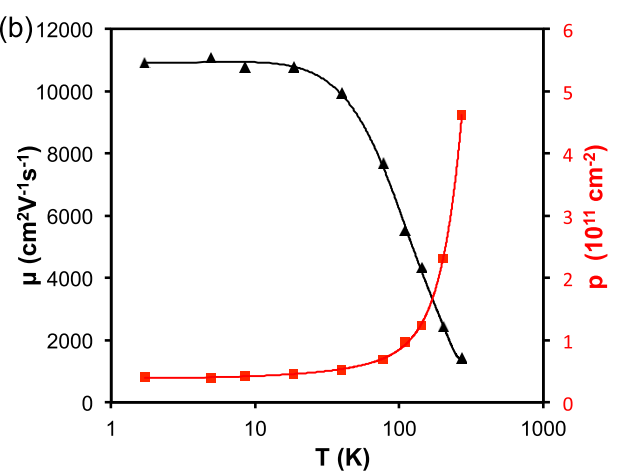

(d)

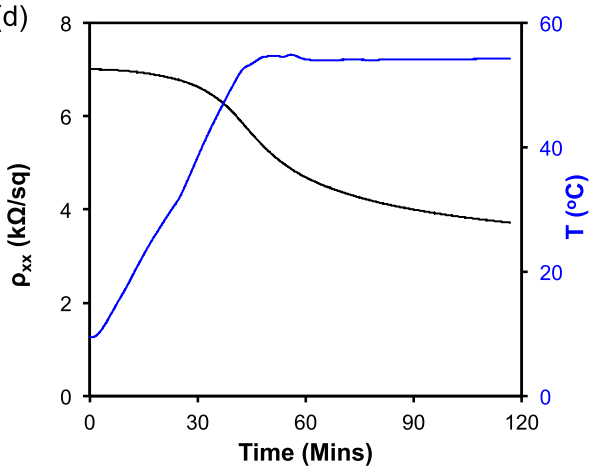

FIG. 2. (a) Overview of the evolving carrier density of $\mathrm{SiC} / \mathrm{G}$ assessed at each process step. ARPES measurements were performed on monolayer $\mathrm{SiC} / \mathrm{G}$ before ${ }^{18}$ and after ${ }^{17}$ aqueousozone processing and annealing (blue circles). Magnetotransport measurements detail the history of a Hall bar device of sample A throughout processing (red squares). (b) Temperature dependent Hall mobility and p-type carrier density of the processed graphene device. Lines are to guide the eye. (c) Monolayer graphene quantum Hall effect, observed at magnetic fields below $2 \mathrm{~T}$, reveals extremely low ptype doping and high mobility after processing. (d) Resistivity as a function of time for the processed $\mathrm{SiC} / \mathrm{G}$ device as the sample is heated to $55^{\circ} \mathrm{C}$. Electron acceptors from the ambient were removed from the graphene surface by mild annealing.
As for the doping mechanism we infer, from the temperature dependence of mobility and Hall coefficient (Figure 2(b)), the presence of thermally activated charge carriers on the measured device. At low temperature, the p-type doping is found to be as low as $4 \times 10^{10}$ holes $\mathrm{cm}^{-2}$. This exceptionally low value indicates a modification after processing to a very homogeneous, charge neutral graphene system. Above $2 \mathrm{~K}$, the carrier density increased significantly due to thermal activation, reaching $\sim 5 \times 10^{11}$ holes $\mathrm{cm}^{-2}$ at room temperature.

In light of the cleaning observations made during the AFM analysis, we considered that the p-type doping effect observed in these samples after processing was caused by the presence of ambient dopants at the surface of the graphene device, enabled by the removal of polymer resist. This effect is similar to that reported in measurements of bare-naked graphene exposed to ambient conditions. ${ }^{1-4,28}$ As a means to validate this hypothesis, we heated the measured device to $55^{\circ} \mathrm{C}$ in a controlled gaseous helium environment, leading to a $47 \%$ decrease in resistivity (Figure 2(d)). Hall measurements after annealing revealed that the device had reverted to n-type doping, with a carrier density of $1.2 \times 10^{12}$ electrons $\mathrm{cm}^{-2}$ (Figure 2(a)). We attributed this modification to labile atmospheric electron acceptors ${ }^{28}$ that were subsequently removed from the graphene surface by annealing.

In summary, we have evaluated the consequences of aqueous-ozone processing and annealing on monolayer graphene Hall bar devices on silicon carbide. We find that devices exposed to the aggressive aqueous-ozone environment became cleaner from post-fabrication organic resist residuals and, significantly, maintained their electronic transport performance in terms of carrier mobility. Quantum Hall effect measurements confirm that transport is dominated by monolayer graphene and, consequently, we rule out intercalation of species at the SiC-graphene interface. This is supported by strong temperature dependence of the carrier mobility indicating the preservation of a strong graphene-substrate interaction. The processed devices exhibit extremely low ptype doping, which is attributed to physisorbed ambient acceptors that gain access to the graphene surface due to the removal of resist residuals. The combination of low carrier density, high mobility, large area clean surfaces, and susceptibility to environmental species suggest this processed graphene system as an ideal candidate for gas sensing applications.

This work was partly supported by the Uppsala University Quality and Renewal program for graphene, the Graphene Flagship (Contract No. CNECT-ICT-604391), Swedish Foundation for Strategic Research (SSF), Linnaeus Centre for Quantum Engineering, Knut and Alice Wallenberg Foundation, Chalmers AoA Nano, and the EMRP project GraphOhm. The EMRP is jointly funded by the EMRP participating countries within EURAMET and the European Union. T.Y. is grateful to Ruth Pearce for useful discussions.

${ }^{1}$ F. Schedin, A. K. Geim, S. V. Morozov, E. W. Hill, P. Blake, M. I. Katsnelson, and K. S. Novoselov, Nat. Mater. 6, 652 (2007).

${ }^{2}$ R. Pearce, T. Iakimov, M. Andersson, L. Hultman, A. L. Spetz, and R. Yakimova, Sens. Actuators, B 155, 451 (2011).

${ }^{3}$ Y. Dan, Y. Lu, N. J. Kybert, Z. Luo, and A. T. C. Johnson, Nano Lett. 9, 1472 (2009).

${ }^{4}$ S. Basu and P. Bhattacharyya, Sens. Actuators, B 173, 1 (2012).

${ }^{5}$ D. A. Bolon and C. O. Kunz, Polym. Eng. Sci. 12, 109 (1972).

${ }^{6}$ J. R. Vig, J. Vac. Sci. Technol., A 3, 1027 (1985).

${ }^{7}$ W. Kern, Handbook of Semiconductor Wafer Cleaning Technology (Noyes Publications, 1993), pp. 111-196.

${ }^{8}$ N. Leconte, J. Moser, P. Ordejon, H. Tao, A. Lherbier, A. Bachtold, F. Alsina, C. S. Torres, J.-C. Charlier, and S. Roche, ACS Nano 4, 4033 (2010).

${ }^{9}$ J. Moser, H. Tao, S. Roche, F. Alzina, C. M. Sotomayor-Torres, and A. Bachtold, Phys. Rev. B 81, 205445 (2010).

${ }^{10}$ H. Tao, J. Moser, F. Alzina, Q. Wang, and C. M. Sotomayor-Torres, J. Phys. Chem. C 115, 18257 (2011). 
${ }^{11}$ E. X. Zhang, A. K. M. Newaz, B. Wang, C. X. Zhang, D. M. Fleetwood, K. I. Bolotin, R. D. Schrimpf, S. T. Pantelides, and M. L. Alles, Appl. Phys. Lett. 101, 121601 (2012).

${ }^{12}$ S. Jandhyala, G. Mordi, B. Lee, G. Lee, C. Floresca, P.-R. Cha, J. Ahn, R. Wallace, Y. Chabal, and M. Kim, ACS Nano 6, 2722 (2012).

${ }^{13}$ F. Alzina, H. Tao, J. Moser, Y. Garcia, A. Bachtold, and C. M. Sotomayor-Torres, Phys. Rev. B 82, 75422 (2010).

${ }^{14}$ J. Moser, A. Barreiro, and A. Bachtold, Appl. Phys. Lett. 91, 163513 (2007).

${ }^{15}$ N. Lindvall, A. Kalabukhov, and A. Yurgens, J. Appl. Phys. 111, 064904 (2012).

${ }^{16}$ A. Nath, A. D. Koehler, G. G. Jernigan, V. D. Wheeler, J. K. Hite, S. C. Hernández, Z. R. Robinson, N. Y. Garces, R. L. Myers-Ward, C. R. Eddy, D. K. Gaskill, and M. V Rao, Appl. Phys. Lett. 104, 224102 (2014).

${ }^{17}$ M. J. Webb, C. Polley, K. Dirscherl, G. Burwell, P. Palmgren, Y. Niu, A. Lundstedt, A. A. Zakharov, O. J. Guy, T. Balasubramanian, R. Yakimova, and H. Grennberg, Appl. Phys. Lett. 105, 081602 (2014).

${ }^{18}$ C. Coletti, C. Riedl, D. S. Lee, B. Krauss, L. Patthey, K. von Klitzing, J. H. Smet, and U. Starke, Phys. Rev. B 81, 235401 (2010).

${ }^{19}$ S. Kopylov, A. Tzalenchuk, S. Kubatkin, and V. I. Fal'ko, Appl. Phys. Lett. 97, 112109 (2010).
${ }^{20}$ R. Yakimova, T. Iakimov, and M. Syväjärvi, "Process for growth of graphene,” U.S. patent PCT/SE2011/050328 (22 March 2012).

${ }^{21}$ A. Tzalenchuk, S. Lara-Avila, A. Kalaboukhov, S. Paolillo, M. Syväjärvi, R. Yakimova, O. Kazakova, T. J. B. M. Janssen, V. Fal'ko, and S. Kubatkin, Nat. Nanotechnol. 5, 186 (2010).

${ }^{22}$ S. Lara-Avila, K. Moth-Poulsen, R. Yakimova, T. Bjørnholm, V. Fal'ko, A. Tzalenchuk, and S. Kubatkin, Adv. Mater. 23, 878 (2011).

${ }^{23}$ T. Yager, A. Lartsev, S. Mahashabde, S. Charpentier, D. Davidovikj, A. V Danilov, R. Yakimova, V. Panchal, O. Kazakova, A. Tzalenchuk, S. LaraAvila, and S. Kubatkin, Nano Lett. 13, 4217 (2013).

${ }^{24}$ T. Yager, A. Lartsev, R. Yakimova, S. Lara-Avila, and S. Kubatkin, "Wafer-Scale Homogeneity of Transport Properties in Epitaxial Graphene on SiC," Carbon (in press); preprint arXiv:1502.02013.

${ }^{25}$ K. S. Novoselov, A. K. Geim, S. V. Morozov, D. Jiang, M. I. Katsnelson, I. V. Grigorieva, S. V. Dubonos, and A. A. Firsov, Nature 438, 197 (2005).

${ }^{26}$ C. Riedl, C. Coletti, T. Iwasaki, A. A. Zakharov, and U. Starke, Phys. Rev. Lett. 103, 246804 (2009).

${ }^{27}$ K. Lee, S. Kim, M. S. Points, T. E. Beechem, T. Ohta, and E. Tutuc, Nano Lett. 11, 3624 (2011).

${ }^{28}$ H. Pinto and A. Markevich, Beilstein J. Nanotechnol. 5, 1842 (2014). 